\title{
Ulcerative balanoposthitis associated with non-syphilitic spirochaetal infection
}

\author{
PETER PIOT,* MARGARET DUNCAN. $\uparrow$ EDDY VAN DYCK.* AND RONALD C BALLARD + \\ From the Departments of Microbiology. ${ }^{*}$ Institute of Tropical Medicine. Antw'erp. Belgium and $\dagger$ South African \\ Institute for Medical Research, Johannesburg. South Africa
}

SUMMARY Ulcerative balanoposthitis associated with non-syphilitic spirochaetal infection was found in $41(12 \%)$ of 344 consecutive men with genital ulcer disease in two clinics in South Africa. All patients with non-syphilitic spirochaetal infection were uncircumcised. Non-syphilitic treponemes were seen in only two of 60 uncircumcised men who had urethritis without genital ulceration. In 14 patients with ulcerative balanoposthitis no cause of genital ulceration could be identified. Most patients presented with large, serpiginous, superficial, foul smelling, and tender ulcers, the base of which was purulent with undermined edges. Non-tender inguinal lymphadenopathy was present in seven of 14 patients. One female sexual partner of a patient with non-syphilitic spirochaetal infection had a vaginal infection with non-syphilitic spirochaetes.

\section{Introduction}

Genital ulcer disease is a common condition in Africa, where genital ulcers are found more often than in the Northern hemisphere.' Several studies have shown that its main cause in Kenya and in southern Africa is chancroid, ${ }^{-4}$ followed by syphilis, herpes genitalis, and other recognised causes of genital ulcer disease. Superficial ulcers are of ten found and can be caused by herpes simplex virus, ${ }^{5}$ anaerobes, ${ }^{6} 7$ Trichomonas vasinalis, ${ }^{x}$ or a fusospirochaetal infection." 10 The latter has been described in older published reports, but recent reports on its occurence and clinical manifestations in the tropics have been rare. ${ }^{10}$ We describe the microbiological and clinical features of patients with extensive superficial genital ulcers, or ulcerative balanoposthitis, associated with nonsyphilitic spirochaetal infection.

\section{Patients and methods}

We saw 159 consecutive black men with genital ulceration at the sexually transmitted disease (STD) clinic of the Leslie Williams Memorial Hospital at Carletonville, Transvaal, and 185 consecutive men with genital ulcer disease at the Hillbrow Hospital, Johannesburg. All patients underwent a standard physical examination and gave details of their sexual history.

Material from the bases of ulcers was collected with a platinum spatula for dark field microscopy. Exudate

Address for reprints: Dr P Piot. Department of Microbiology. Institute of Tropical Medicinc. Nationalestratal 155. B-2(Xi) Antucrpen. Belgium from the ulcer base was subsequently collected with calcium alginate urethral swabs, which were immediately used to inoculate a selective isolation medium for Hacmophilus ducreyi $i^{3}$ and a transport medium for herpes simplex virus and Chlamydia trachomatis. $C$ trachomatis was cultured on cycloheximide treated McCoy cells, and herpes simplex virus on human embryo foreskin fibroblasts. Serological tests for syphilis included a rapid plasma reagin (RPR) test and a fluorescent treponemal antibody absorbed (FTA-ABS) assay (Wellcome), and chlamydial serum antibodies were identified by a modified microimmunofluorescence test. ${ }^{\prime \prime}$

\section{Results}

Of 344 patients with genital ulcer disease, $41(12 \%)$ had purulent erosive balanoposthitis. In all instances this syndrome was associated with the presence of large numbers of mixed spirochaetes observed on dark field examination. These spirochaetes were of different sizes, usually with coils more open than those of Treponema pallidum. The movements of these bacteria were variable and were often eel like rather than the typical cork screw like movement of $T$ pallidum. The spirochaetes were invariably accompanied by large numbers of bacteria of various shapes and also by leucocytes. Two spirochaetes were found on dark ground examination of scrapings from the subpreputial sac of 60 uncircumcised black men with urethritis seen at the Carletonville STD clinic.

We isolated $H$ ducreyi from nine $(22 \%)$ of the 41 patients, herpes simplex virus from four $(10 \%)$ herpes 
virus and $H$ ducreyi from one, $C$ trachomatis from one, and $H$ ducreyi and $C$ trachomatis from two. In hine cases a diagnosis of primary syphilis was made based on positive serological tests, and one patient had both syphilis and chancroid. Thus we identified an additional known cause of genital ulcer disease in a total of $27(66 \%)$ of 41 patients with non-syphilitic spirochaetal infection.

We present in detail the features of the 14 patients without an identifiable cause of genital ulcer disease. Their mean age was 23 (range 21 to 27 ) years. The presumed mean incubation period of the genital lesions was 15.7 (range two to 60 ) days, and the mean duration of illness before presentation was 12 (range four to 17) days. The illness started with multiple small superficial ulcers (fig (a)). Most patients, however, presented with large serpiginous and superficial ulcers, which resembled those of ulcerative balanoposthitis (fig (b)). The base of the lesion was indurated in 13 cases, purulent in 13, and had undermined edges in 11. The discharge was usually foul smelling. In nine of the 14 patients the ulcers were tender. We found all cases of non-syphilitic spirochaetal infection in uncircumcised men. In contrast, non-syphilitic spirochaetal infection had not been detected among 120 consecutive circumcised patients examined at the Carletonville STD clinic in 1984. Two of the 14 patients with no other identifiable cause of genital ulcer disease presented with phimosis. Half the patients had inguinal lymphadenopathy, which was bilateral. in two cases. These lymph nodes were not tender, and their average diameter was 21 $\mathrm{mm}$ (range 5 to $52 \mathrm{~mm}$ ).

We examined one female sexual partner of a man with non-syphilitic spirochaete associated genital ulcer disease. She presented with a profuse purulent vaginal discharge and superficial lesions of the vaginal wall. Numerous non-syphilitic spirochaetes were seen on dark field examination of the vaginal fluid. Serological tests for syphilis gave negative results.

\section{Discussion}

In the population studied, extensive superficial ulcers without an identifiable cause were appreciably associated with the presence of non-syphilitic spirochaetes on dark field microscopy of ulcer material in the absence of positive serological tests for syphilis at presentation and at follow up examinations. No attempts were made to identify these spirochaetes, but their characteristic eel like movements and few coils suggested that they belonged to the non-syphilitic treponemes or the Borrelia group. This group includes non-pathogenic ill-defined species, such as $T$ (Borrelia) refrigens. $T$ phagedenis and $T$ balanitidis, which have been identified on the genitalia, and $T$ (Borrelia) vincenti, as found in the fusospirochaetal association. ${ }^{12}$ The presence of

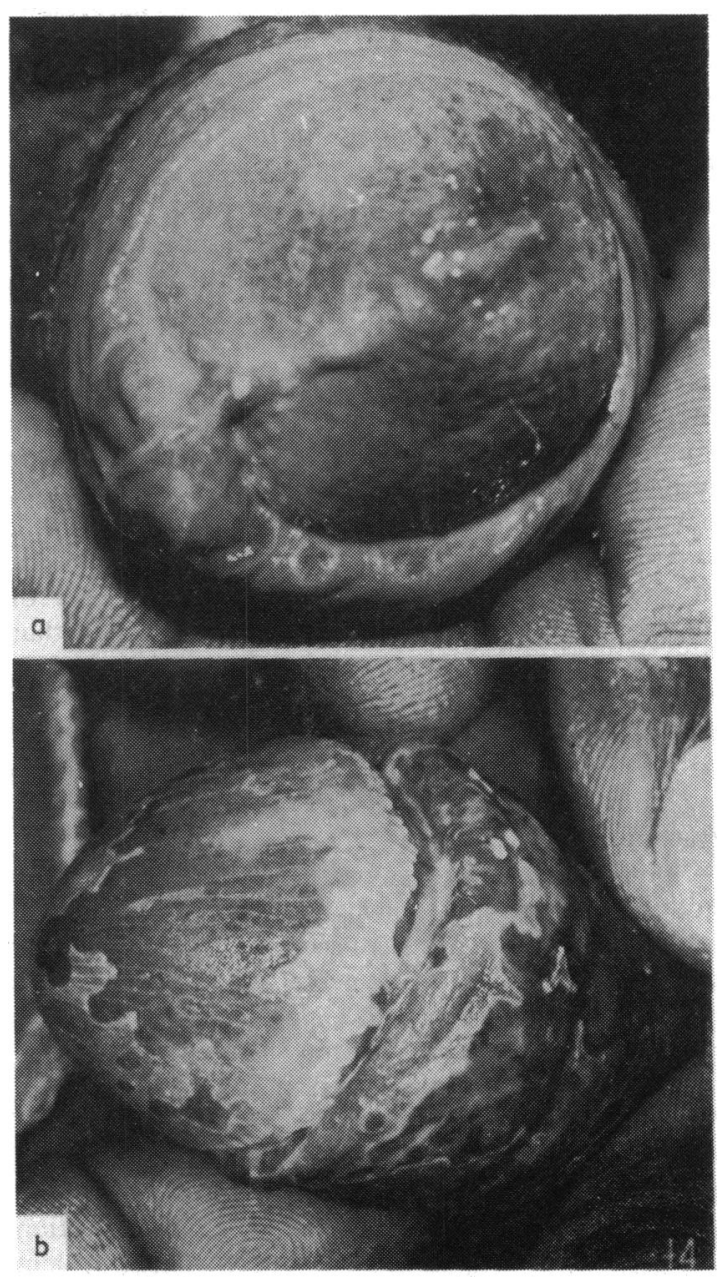

FIGURE Ulcerative balanoposthitis associated with nontreponemal spirochactes (a) two days after onset of disease. (b) seven days after onset of disease.

numerous bacteria on dark ground examination and of an offensive smelling discharge suggested that other bacteria, such as obligate anaerobes, were present in spirochaete associated ulcerative balanoposthitis. Such spirochaetes or associated bacteria were not seen in uncircumcised controls, though small numbers would not be detected by microscopy.

Similar forms of ulcerative balanoposthitis have been attributed to fusospirochaetal association ${ }^{8}$ and to anaerobic infection, usually consisting of a mixture of non-spore-forming anaerobes, such as Bacteroides spp and Fusobacterium spp. ${ }^{6}$ A non-erosive balanoposthitis characterised by mild inflammation and fishy smelling subpreputial discharge has been reported for a mixed infection of Bacteroides spp and Gardnerella vaginalis. ${ }^{13}$ All these variants of 
balanoposthitis may be caused by single or mixed infections with anaerobes, and the severity of a lesion is possibly determined either by the type of microorganisms implicated in its pathogenesis or by the duration of disease. As in our series, it is striking that all reports stress that men with spirochaetal or anaerobic balanoposthitis were uncircumcised. Poor hygiene is likely to create an anaerobic environment under the prepuce, thus favouring the multiplication of anaerobes and other bacteria. As is the case in bacterial vaginosis, ${ }^{14}$ the quantity (rather than the mere presence) of a micro-organism or the combination of different micro-organisms may be important in the pathogenesis of ulcerative balanoposthitis.

As anaerobic species that dominate the normal flora of the oropharynx have been found in cases of anaerobic balanoposthitis, Cree et al suggested that the condition may be acquired from the oral cavity as a result of digital or orogenital transmission. ${ }^{6}$ The finding of similar spirochaetes in a female sexual partner with vaginitis, however, suggests that nonsyphilitic spirochaetal infection may be sexually transmitted.

Spirochaetal ulcerative balanoposthitis is probably not uncommon in tropical areas, as shown by an incidence of $12 \%$ among consecutive men with genital ulcers in the present series, and $8 \%$ of 113 consecutive cases of balanoposthitis in Calcutta, India. ${ }^{10}$ In one STD clinic in the United Kingdom erosive balanoposthitis was diagnosed in $30 \%$ of all cases of balanitis. $^{6}$

A presumptive clinical diagnosis of ulcerative balanoposthitis associated with spirochaetes can be made in the presence of an extensive tender ulceration of the glans accompanied by a foul smelling purulent discharge. The finding of non-treponemal spirochaetes associated with large numbers of bacteria on dark field examination confirms the diagnosis. Particularly in tropical areas, where this condition may be common, these spirochaetes may be a source of confusion with $T$ pallidum. Treatment with penicillins, and perhaps with metronidazole, ${ }^{6}$ may prevent progression to phagedaenal complications.
This study was supported in part by the South African Medical Research Council. We also thank Ms HG Fehler for excellent technical help and Dr P Lowe and the staff of the Leslie Williams' Memorial Hospital for providing access to patients.

\section{References}

1. Piot $\mathbf{P}$, Holmes KK. Sexually transmitted diseases. In: Warren KS, Mahmoud AFF, eds. Tropical and geographical medicine. New York: McGraw Hill, 1984:844-62.

2. Nsanze H, Fast MV, D'Costa LJ, Tukei P, Curran J, Ronald A. Genital ulcers in Kenya: clinical and laboratory study. British Journal of Veneral Diseases 1981;57:378-81.

3. Duncan MO, Bilgeri YR, Fehler HG, Ballard RC. The diagnosis of sexually acquired genital ulcerations in black patients in Johannesburg. South African Journal of Sexually Transmitted Diseases 1981;1:20-3.

4. Meheus A, Van Dyck E, Ursi JP, Ballard RC, Piot P. Etiology of genital ulcerations in Swaziland. Sex Transm Dis 1983;10:335.

5. Peutherer JF, Smith IW, Robertson DHH. Necrotising balanitis due to a generalised primary infection with herpes simplex virus type 2. British Journal of Venereal Diseases 1979;55:48-51.

6. Cree GE, Willis AT, Phillips KD, Brazier JS. Anaerobic balanoposthitis. Br Med J 1982;284:859-60.

7. Corbus BC, Harris FG. Erosive and gangrenous balanitis. The fourth venereal disease. JAMA 1909;52:1474-7.

8. Michalowski R. Balano-posthites à trichomonas. A propos de 16 observations. Ann Dermatol Venereol 1981;108:731-8.

9. Brams J, Pilot I, Davis DJ. Studies of fusiform bacilli and spirochetes. II. Their occurrence in normal preputial secretions and in erosive and grangrenous balanitis.' $J$ Infect Dis 1923;32:159-66.

10. Chakraborty AK. Clinico-pathological study of balanoposthitis in male. Indian J Dermatol 1982;27:105-8.

11. Treharne JD, Darougar S, Jones BR. Modification of the microimmunofluorescence test to provide a routine serodiagnostic test for chlamydial infection. $J$ Clin Pathol 1977;30:510-7.

12. Krieg NR, Holt JG, eds. Bergey's manual of systematic bacteriology. Baltimore: Williams \& Wilkins, 1984; vol 1.

13. Kinghorn GR, Jones BM, Chowdhury FH, Geary I. Balanoposthitis associated with Gardnerella vaginalis infection in men.British Journal of Venereal Diseases 1982;58:127-9.

14. Spiegel CA, Amsel R, Eschenback D, Schoenknecht F, Holmes KK. Anaerobic bacteria in non-specific vaginitis. $N$ Engl J Med 1980;303:601-5. 